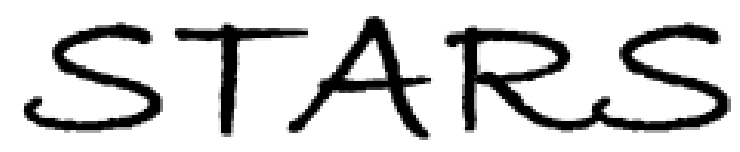

University of Central Florida

STARS

$1-1-2012$

\title{
Cascaded field enhancement in plasmon resonant dimer nanoantennas compatible with two-dimensional nanofabrication methods
}

Seyfollah Toroghi

University of Central Florida

Pieter G. Kik

University of Central Florida

Find similar works at: https://stars.library.ucf.edu/facultybib2010

University of Central Florida Libraries http://library.ucf.edu

This Article is brought to you for free and open access by the Faculty Bibliography at STARS. It has been accepted for inclusion in Faculty Bibliography 2010s by an authorized administrator of STARS. For more information, please contact STARS@ucf.edu.

\section{Recommended Citation}

Toroghi, Seyfollah and Kik, Pieter G., "Cascaded field enhancement in plasmon resonant dimer nanoantennas compatible with two-dimensional nanofabrication methods" (2012). Faculty Bibliography 2010s. 3396.

https://stars.library.ucf.edu/facultybib2010/3396

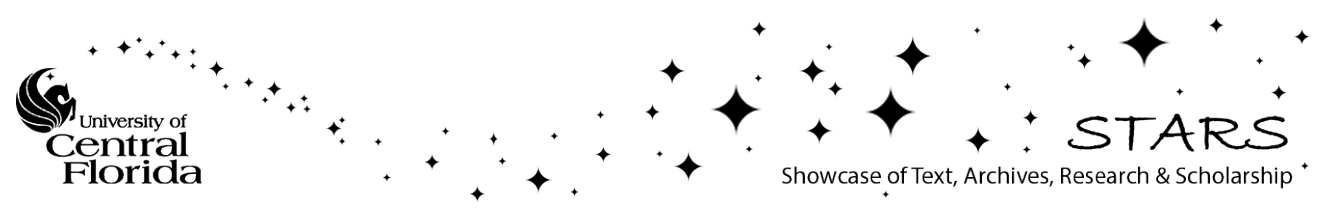




\section{Cascaded field enhancement in plasmon resonant dimer nanoantennas compatible with two-dimensional nanofabrication methods}

Cite as: Appl. Phys. Lett. 101, 013116 (2012); https://doi.org/10.1063/1.4733329

Submitted: 27 April 2012 . Accepted: 20 June 2012 . Published Online: 05 July 2012

Seyfollah Toroghi, and Pieter G. Kik

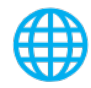

\section{ARTICLES YOU MAY BE INTERESTED IN}

Cascaded plasmon resonant field enhancement in nanoparticle dimers in the point dipole limit Applied Physics Letters 100, 183105 (2012); https://doi.org/10.1063/1.4707159

Collective plasmon modes in a compositionally asymmetric nanoparticle dimer AIP Advances 1, 032134 (2011); https://doi.org/10.1063/1.3628346

Plasmon resonance enhanced temperature-dependent photoluminescence of Si-V centers in diamond

Applied Physics Letters 107, 211905 (2015); https://doi.org/10.1063/1.4936332

Applied Physics Reviews Now accepting original research 


\title{
Cascaded field enhancement in plasmon resonant dimer nanoantennas compatible with two-dimensional nanofabrication methods
}

\author{
Seyfollah Toroghi and Pieter G. Kik ${ }^{\text {a),b) }}$ \\ CREOL, The College of Optics and Photonics, University of Central Florida, 4000 Central Florida Blvd, \\ Orlando, Florida 32816, USA
}

(Received 27 April 2012; accepted 20 June 2012; published online 5 July 2012)

\begin{abstract}
Cascaded field enhancement is demonstrated in asymmetric plasmon resonant dimer nanoantennas consisting of shape-tuned ellipsoidal nanoparticles. The nanoparticles that make up the dimer have identical thickness, suggesting that the presented approach can be used to design cascaded dimer antennas compatible with standard two-dimensional top-down nanofabrication tools such as electron beam lithography and nano-imprint lithography. Cascaded excitation is achieved by modification of the in-plane particle aspect ratios in a way that keeps the resonance frequency of the individual particles fixed while significantly changing their polarizability. The achievable field enhancement is evaluated as a function of the particle volume ratio and spacing. (C) 2012 American Institute of Physics. [http://dx.doi.org/10.1063/1.4733329]
\end{abstract}

Coupled plasmon resonances have attracted considerable interest due to their strong field enhancement and resonance shifts which enable them to be used in applications such as surface-enhanced Raman spectroscopy (SERS), ${ }^{1-3}$ enhanced optical nonlinear response, ${ }^{4-6}$ enhanced spontaneous emission, ${ }^{7}$ and molecular rulers. ${ }^{8}$ Recently, a special class of coupled plasmon resonances called cascaded plasmon resonances was shown to provide even stronger field enhancement factors. ${ }^{1,9}$ In cascaded plasmon resonance, near-field coupling of nanoparticles that have the same plasmon resonance frequency but significantly different volume can lead to multiplicative field enhancement in which the smaller of the particles is excited predominantly by the enhanced near-fields of the larger particle. The simplest system that produces this effect is shown in Fig. 1(a), consisting of an asymmetric nanosphere dimer. However, it is difficult to fabricate such cascaded nanosphere structures with standard top-down nanofabrication tools such as electron beam lithography or nano-imprint lithography since these methods typically produce structures that have a fixed thickness, determined for example by a thin film deposition step in the process. Under these conditions, changing the volume of nanoparticles requires a modification of the lateral size of and therefore the aspect ratio of the particles, which is known to cause significant resonance shifts. ${ }^{10,11}$ This implies that one of the main requirements for cascaded plasmon resonance cannot be satisfied in top-down nanofabricated structures, posing a significant challenge to the development of technologies that utilize cascaded plasmon resonances. In the present work we demonstrate that cascaded plasmon resonance can be achieved in shape-tuned dimer antennas with identical maximum thickness (Figs. 1(b)-1(d)) by modifying the in-plane particle aspect ratio to correct for size induced resonance shifts.

In order to study the feasibility of designing cascaded plasmon resonances using nanoparticles with identical thick-

\footnotetext{
a) Also at Physics Department, University of Central Florida, 4000 Central Florida Blvd, Orlando, Florida 32816, USA.

b)Electronic mail: kik@creol.ucf.edu.
}

ness, we consider dimer antennas composed of ellipsoidal particles. Ellipsoidal metal nanoparticles support dipolar localized plasmon resonances with a localized plasmon resonance frequency that is affected by the particle aspect ratio. The plasmon resonance frequency of ellipsoids can be obtained from the expression for the polarizability. In the quasi-electrostatic approximation, the polarizability of an ellipsoid with axes $a, b$, and $c$, illuminated by a plane wave polarized along the $a$ direction, is given by ${ }^{11}$

$$
\alpha_{a}=V \frac{\varepsilon_{m}-\varepsilon_{h}}{\varepsilon_{h}+L_{a}\left(\varepsilon_{m}-\varepsilon_{h}\right)},
$$

where $\varepsilon_{m}$ and $\varepsilon_{h}$ are the dielectric function of the metal and the host material, respectively, and $V$ is the volume of the ellipsoidal particle given by $(a b c \pi / 6)$. Equation (1) assumes that the particle dipole moment relates to the incident field according to $p=\varepsilon_{0} \varepsilon_{h} \alpha E$ with $\varepsilon_{0}$ the permittivity of vacuum. $L_{a}$ is the shape factor given by ${ }^{11}$

$$
L_{a}=\frac{a b c}{2} \int_{0}^{\infty}\left(a^{2}+q\right)^{-\frac{3}{2}}\left(b^{2}+q\right)^{-\frac{1}{2}}\left(c^{2}+q\right)^{-\frac{1}{2}} d q .
$$

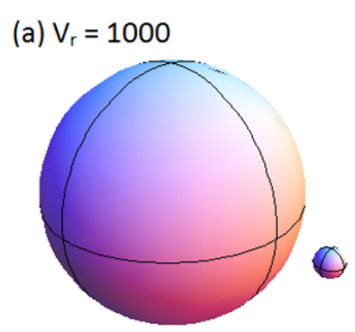

(b) $V_{r}=10$

(c) $V_{r}=100$

(d) $V_{r}=1000$
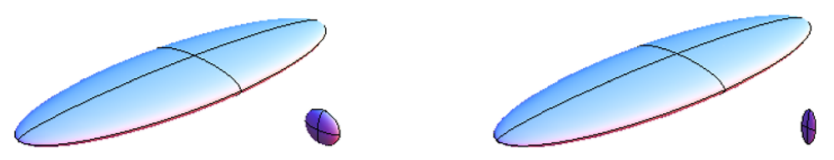

FIG. 1. (a) Cascaded nanosphere dimer with volume ratio of $V_{r}=1000$ and shape-tuned cascaded nanoparticle dimers with volume ratios of (b) 10, (c) 100, and (d) 1000 . 
Shape factors for excitation with the incident field along the $b$ and $c$ directions can be obtained by exchanging the position of $a$ with that of $b$ or $c$ in Eq. (2), respectively. In addition, the electric field inside the ellipsoid, in the quasielectrostatic approximation, is given by ${ }^{11}$

$$
E_{i n}=\frac{\varepsilon_{h}}{\varepsilon_{h}+L_{a}\left(\varepsilon_{m}-\varepsilon_{h}\right)} E_{0}
$$

where $E_{0}$ is the amplitude of the electric field of the incident light. The corresponding maximum external field enhancement is given by $E_{\text {out }}=\left(\varepsilon_{m} / \varepsilon_{h}\right) E_{\text {in }}$. The dipolar plasmon resonance occurs when the real part of the denominator of the polarizability (Eq. (1)) is zero, corresponding to the resonance condition $\varepsilon_{m}{ }^{\prime}=-R \varepsilon_{h}$, where $\varepsilon_{m}{ }^{\prime}$ is the real part of the dielectric function of the metal and $R=\left(1 / L_{a}-1\right)$. For a sphere $L_{a}=1 / 3$ leading to the well-known resonance requirement $\varepsilon_{m}{ }^{\prime}=-2 \varepsilon_{h}$. The imaginary part of the host dielectric function is assumed to be negligible. Figure 2 shows a contour plot of calculated resonance conditions for a large range of particle shapes. The axis lengths are scaled with respect to the axis that is aligned with the polarization of the incident light. For readability of the graph, the logarithms of the axis ratios and the resonance condition are displayed. Since the resonance condition is not affected by an interchange of axes $b$ and $c$ in the quasi-electrostatic limit, all unique shapes and resonance conditions with axis ratios between 0.1 and 10 are represented by the data in the upper left side of the graph. Six example shapes and their corresponding aspect ratios have been included and labeled with roman numerals. Figure 2 demonstrates that it is possible to design nanoparticles with different shape but identical resonance frequency: by choosing shapes that lie on one of the contour lines and scaling axis $b$ or $c$ to the desired thickness, we obtain shapes with different volume but the same resonance frequency, which is

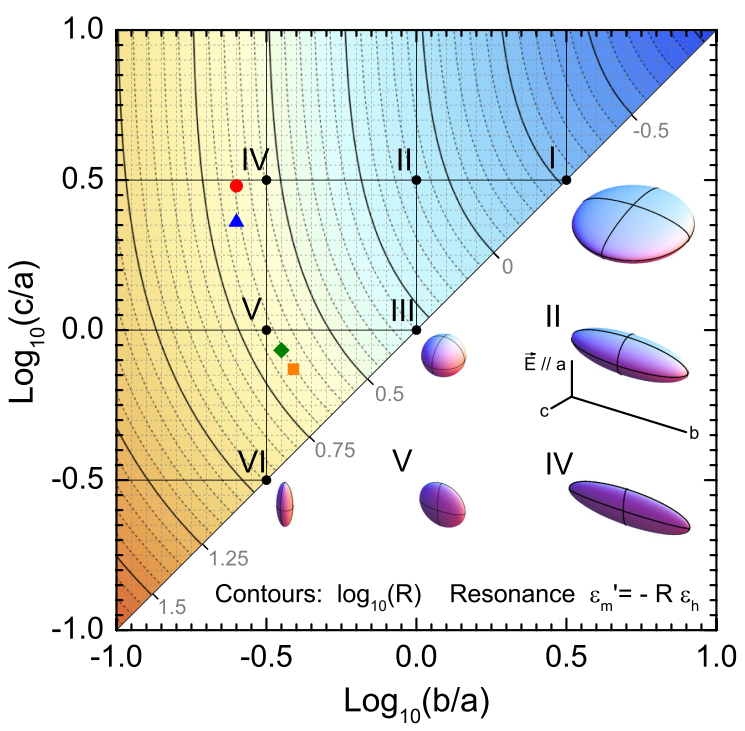

FIG. 2. Shape dependent dipolar resonance conditions for ellipsoidal nanoparticles with axis lengths $a, b$, and $c$, illuminated by an electromagnetic wave polarized along the $a$-axis. Contour lines represent the logarithm of the resonance prefactor $\mathrm{R}$. The insets show ellipsoids with the axis ratios indicated by the roman numerals. The symbols indicate the aspect ratios used in the cascaded dimer antennas shown in Fig. 1. one of the key requirements for achieving cascaded plasmon resonance.

In this study we consider dimer nanoantennas consisting of silver nanoellipsoids embedded in a host material with $\varepsilon_{\mathrm{h}}=2.25$. The thickness of all ellipsoids was held constant at $5 \mathrm{~nm}$. The largest particle considered in this study has axis lengths $(a, b, c)$ given by $(20 \mathrm{~nm}, 5 \mathrm{~nm}, 60 \mathrm{~nm})$. The corresponding aspect ratio is indicated by the red circle in Fig. 2. From the contour line or through numerical evaluation of Eq. (1) it can be shown that this shape is expected to exhibit a dipolar resonance when $\varepsilon_{m}{ }^{\prime}=-4.42 \varepsilon_{h}$. However the finite size of the ellipsoid is expected to cause small resonance shifts when taking into account retardation effects. To determine the actual resonance wavelength of this ellipsoid, frequency domain electromagnetic simulations ${ }^{12}$ were carried out. The ellipsoidal Ag particle was illuminated with a plane wave with electric field amplitude $E_{0}$, propagating in the direction corresponding to the thickness of the particle ("normal incidence") and polarized along the $a$ axis of the particle. Figure 3 shows the resulting frequency dependent electric field amplitude at the center of the ellipsoid, relative to the incident wave amplitude. The corresponding particle shape is shown as an inset. A clear plasmon resonance is observed at $\lambda=508 \mathrm{~nm}$, corresponding to a resonance condition $\varepsilon_{m}{ }^{\prime}=-4.5 \varepsilon_{h}$, close to the analytically predicted result. Three additional particle shapes were selected with a volume that is, respectively, a factor 10, 100, and 1000 smaller than that of the largest particle. The corresponding shapes are $(12.8 \mathrm{~nm}, 5 \mathrm{~nm}, 9.4 \mathrm{~nm}),(5.8 \mathrm{~nm}, 2.1 \mathrm{~nm}, 5 \mathrm{~nm})$, and $(2.2 \mathrm{~nm}$, $0.56 \mathrm{~nm}, 5 \mathrm{~nm}$ ) as marked in Fig. 2, respectively, by a solid square, diamond, and triangle. Note that these shapes all lie close to the contour line $\log _{10}(\mathrm{R})=0.65$, corresponding to a resonance condition $\varepsilon_{m}{ }^{\prime}=-4.5 \varepsilon_{h}$. The corresponding simulated internal field enhancement spectra are included in Fig. 3 , all showing a resonance wavelength of $508 \pm 1 \mathrm{~nm}$. The largest simulated internal field enhancement is 35 , corresponding to the analytically predicted result within $1.5 \%$.

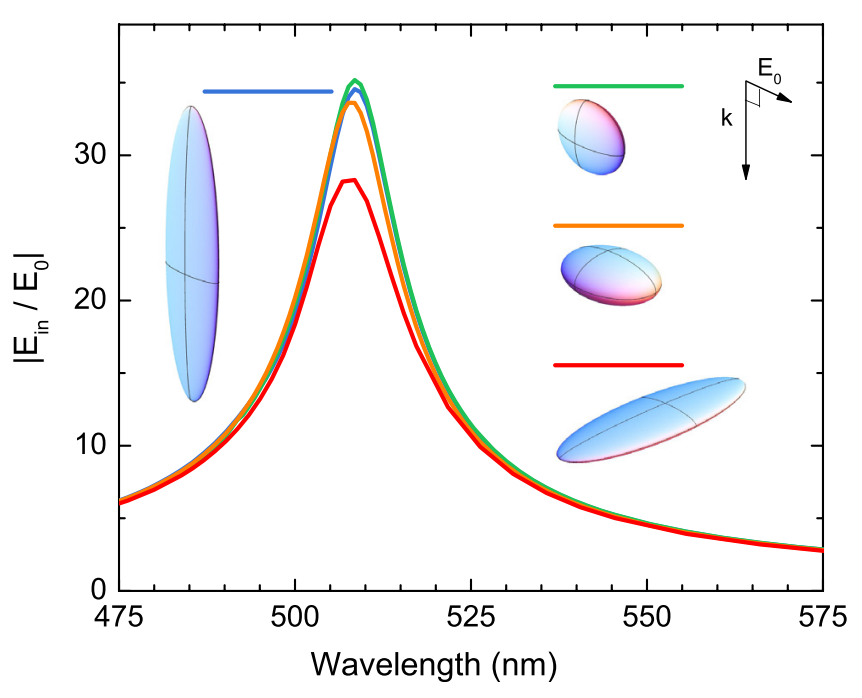

FIG. 3. Internal field enhancement spectra of isolated silver nanoparticles in a host with dielectric function $\varepsilon_{h}=2.25$ with axes $(a, b, c)$ given by $(2.2 \mathrm{~nm}$, $0.56 \mathrm{~nm}, 5 \mathrm{~nm}),(5.8 \mathrm{~nm}, 2.1 \mathrm{~nm}, 5 \mathrm{~nm}),(12.8 \mathrm{~nm}, 5 \mathrm{~nm}, 9.4 \mathrm{~nm})$, and $(20 \mathrm{~nm}$, $5 \mathrm{~nm}, 60 \mathrm{~nm}$ ). The legend shows the corresponding shapes (not to scale). 
To investigate the development of cascaded plasmon resonances in shape-tuned dimer antennas, dimers with three different volume ratios were considered based on the four ellipsoids shown in Fig. 3. The corresponding antenna structures are shown in Figs. 1(b)-1(d) for a center-to-center spacing of $d=25 \mathrm{~nm}$. Figure 4(a) shows the simulated internal field enhancement at the center of the small nanoparticle for the three different dimer nanoantennas with $d=40 \mathrm{~nm}$. The corresponding volume ratios are 10 (orange line), 100 (green line), and 1000 (blue line). The internal field enhancement of the largest isolated nanoparticle (red line) is included for comparison, corresponding to the red curve in Fig. 3. The maximum internal field enhancement factors for the coupled dimer antennas occur at a wavelength that is slightly red-shifted compared to dipole resonance of the isolated particles, indicative of relatively weak inter-particle interaction. The field enhancement factors are slightly larger than observed for the isolated particles in Fig. 3. Figure 4(b) shows the results for the same particle combinations for a smaller center-to-center spacing of $d=25 \mathrm{~nm}$. Several notable differences are observed. First, the dimer with a relatively small volume ratio of 10 shows a significant red-shift, indicative of increased mutual interaction. This effect is not observed for the two dimers with larger volume ratios, which is attributed to the lower polarizability of the smaller particles used. Second, the obtained field enhancement factors are significantly larger than in Fig. 4(a), indicating the appearance of significant cascading. For the largest two volume ratios (smallest particles), the peak enhancement factors are similar despite the very different particle sizes. This is an expected result in the case of weak mutual interaction and corresponds to a coupling regime called "multiplicative cascading." 13 Figure 4(c) shows the corresponding results for a spacing of $d=17 \mathrm{~nm}$. The spectrum for a volume ratio of 10 could not be included as the small center-to-center spacing would lead to overlapping particles. The peak field enhancement of the dimer with volume ratio 100 exhibits a significant redshift compared to the isolated particle

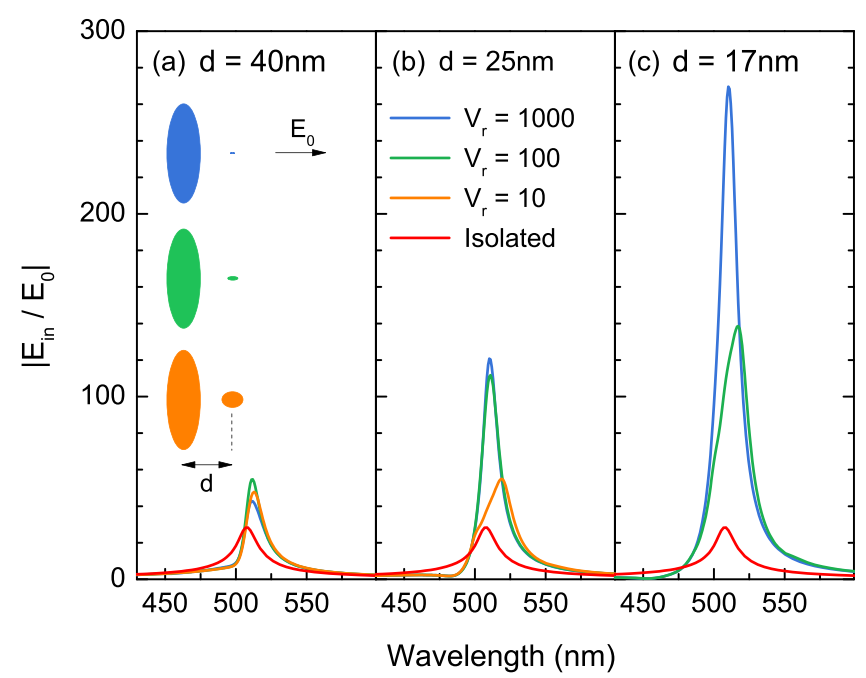

FIG. 4. Internal field enhancement spectra for the smaller nanoparticle in asymmetric silver dimer nanoantennas at a center-to-center spacing of (a) $40 \mathrm{~nm}$, (b) $25 \mathrm{~nm}$, and (c) $17 \mathrm{~nm}$ shown for volume ratios $V_{\mathrm{r}}$ of 10, 100, and 1000. The internal field enhancement spectrum of the isolated large nanoparticle with fixed dimensions $(20 \mathrm{~nm}, 5 \mathrm{~nm}, 60 \mathrm{~nm})$ is included for comparison. response, indicative of stronger inter-particle interaction at this smaller spacing. The dimer with a volume ratio of 1000 shows only a small peak shift of $\sim 2 \mathrm{~nm}$ and a strongly improved field enhancement with a peak value of 270, a factor 9.5 larger than that for the larger nanoparticle.

The field enhancement factors observed in Fig. 4 do not represent the maximum achievable values. Based on an analysis similar to that given in Ref. 13, it can be shown that the maximum field enhancement, i.e., the "ultimate cascading limit," for internal field enhancement of coupled ellipsoidal dimers in the quasi-electrostatic limit occurs at the localized plasmon resonance frequency $\omega_{\mathrm{LSP}}$ and is given by

$$
\left|g_{i n, U C L}\right|=\left|\frac{E_{i n}}{E_{0}}\right|=\frac{Q_{L S P} \sqrt{1+Q_{L S P}^{2}}}{\left(1-L_{a}\right) L_{a}},
$$

where $\mathrm{Q}_{\mathrm{LSP}}=\operatorname{Re}\left[\varepsilon_{\mathrm{m}}\left(\omega_{\mathrm{LSP}}\right)\right] / \operatorname{Im}\left[\varepsilon_{\mathrm{m}}\left(\omega_{\mathrm{LSP}}\right)\right]$. The ultimate external field enhancement factor follows from $\left|\mathrm{g}_{\text {out }, \mathrm{UCL}}\right|$ $\approx\left|1-1 / L_{a}\right| \times\left|\mathrm{g}_{\mathrm{in}, \mathrm{UCL}}\right|$.

Equation (4) predicts a maximum internal field enhancement factor of $5.7 \times 10^{3}$, significantly larger than the results shown in Fig. 4(c)), and a maximum external field enhancement factor of $2.6 \times 10^{4}$. While mathematically and in numerical simulation this result can be achieved, it would require a reduction of the smallest nanoparticle width to below the atomic radius, making such structures experimentally unfeasible. Nevertheless, the results in Fig. 4 unambiguously demonstrate that a significant degree of cascaded field enhancement can be achieved in shape tuned dimer antennas with fixed thickness and large volume ratios. Such structures could be fabricated, e.g., using electron beam lithography, within the resolution limits of the technique. Experimental demonstrations of these effects will need to take into account retardation effects including multipolar excitations, surface scattering for small particle sizes, the asymmetric environment due to the presence of a substrate, and deviations from the ideal ellipsoidal shapes that were considered in the present study. For example, the presence of a high-index substrate is expected to introduce spectral shifts due to a change in the local dielectric environment and could enable the excitation of multipolar modes not present in the current study due to a modification of the symmetry of the local environment. Depending on the importance of these experimental factors on the ultimate field enhancement factors, the presented dimer antenna design could become a basic building block in top-down nanofabricated cascaded plasmon resonant devices.

In summary, it was demonstrated through numerical simulation that cascaded plasmon resonances can be achieved in dimer nanoantennas consisting of near-field coupled ellipsoidal particles with identical thickness. A shape optimization method was presented that may be used to design asymmetric dimers consisting of nanoparticles with an identical plasmon resonance frequency. This approach could lead to cascaded antenna designs that are compatible with common top-down nanofabrication methods.

This work was supported by the National Science Foundation (CAREER Award No. ECCS-0644228). 
${ }^{1}$ K. Li, M. I. Stockman, and D. J. Bergman, Phys. Rev. Lett. 91(22), 227402 (2003).

${ }^{2}$ C. E. Talley, J. B. Jackson, C. Oubre, N. K. Grady, C. W. Hollars, S. M. Lane, T. R. Huser, P. Nordlander, and N. J. Halas, Nano Lett. 5(8), 1569-1574 (2005).

${ }^{3}$ V. G. Kravets, G. Zoriniants, C. P. Burrows, F. Schedin, C. Casiraghi, P. Klar, A. K. Geim, W. L. Barnes, and A. N. Grigorenko, Phys. Rev. Lett. 105(24), 246806 (2010).

${ }^{4}$ K. Li, M. I. Stockman, and D. J. Bergman, Phys. Rev. B 72(15), 153401 (2005).

${ }^{5}$ M. Danckwerts and L. Novotny, Phys. Rev. Lett. 98(2), 026104 (2007).

${ }^{6}$ S. Toroghi and P. G. Kik, Phys. Rev. B 85(4), 045432 (2012).
${ }^{7}$ A. Mohammadi, V. Sandoghdar, and M. Agio, New J. Phys. 10, 105015 (2008).

${ }^{8}$ C. Sonnichsen, B. M. Reinhard, J. Liphardt, and A. P. Alivisatos, Nat. Biotechnol. 23(6), 741-745 (2005).

${ }^{9}$ G. Sun, J. B. Khurgin, and A. Bratkovsky, Phys. Rev. B 84(4), 045415 (2011).

${ }^{10}$ K. L. Kelly, E. Coronado, L. L. Zhao, and G. C. Schatz, J. Phys. Chem. B 107(3), 668-677 (2003).

${ }^{11}$ C. F. Bohren and D. R. Huffman, Absorption and Scattering of Light by Small Particles (Wiley, New York, 1983).

${ }^{12}$ Microwave Studio, Computer Simulation Technology, Darmstadt, Germany, 2011.

${ }^{13}$ S. Toroghi and P. G. Kik, Appl. Phys. Lett. 100(18), 183105 (2012). 